\title{
Frequency Equation of Flexural Vibrating Cantilever Beam Considering the Rotary Inertial Moment of an Attached Mass
}

\author{
Binghui Wang, ${ }^{1}$ Zhihua Wang, ${ }^{2}$ and Xi Zuo ${ }^{3}$ \\ ${ }^{1}$ School of Architecture and Civil Engineering, Jiangsu University of Science and Technology, 2 Mengxi Road, Zhenjiang, Jiangsu, China \\ ${ }^{2}$ Institute of Geotechnical Engineering, Nanjing Tech University, 200 North Zhongshan Road, Nanjing, China \\ ${ }^{3}$ Institute of Architectural Engineering, Jinling Institute of Technology, Nanjing, China \\ Correspondence should be addressed to Zhihua Wang; wzhnjut@163.com
}

Received 13 December 2016; Accepted 5 February 2017; Published 22 February 2017

Academic Editor: Rahmat Ellahi

Copyright (C) 2017 Binghui Wang et al. This is an open access article distributed under the Creative Commons Attribution License, which permits unrestricted use, distribution, and reproduction in any medium, provided the original work is properly cited.

\begin{abstract}
The major goal of this paper is to address the derivation of the frequency equation of flexural vibrating cantilever beam considering the bending moment generated by an additional mass at the free end of beam, not just the shear force. It is a transcendental equation with two unambiguous physical meaning parameters. And the influence of the two parameters on the characteristics of frequency and shape mode was made. The results show that the inertial moment of the mass has the significant effect on the natural frequency and the shape mode. And it is more reasonable using this frequency equation to analyze vibration and measure modulus.
\end{abstract}

\section{Introduction}

The cantilever beam is a simple structure, and it is an important simplified model for many engineering problem in the fields of mechanical engineering, civil engineering, and so forth. However, there is a vast number of papers concerned with the determination of the eigenfrequencies of the cantilever beam subject to various boundary conditions, which can be found in the classic book [1]. And more and more issues of cantilever beam with complicated boundary conditions [2] or external load conditions [3,4] have been studied by theoretical deduction [5] or numerical method [6], or it is application to determine Young's modulus [7].

The cantilever beam model is also used in geotechnical earthquake engineering as a simplified model [8] for the ground responses due to earthquake. It is also a basic principle of measuring the soil's dynamic shear modulus which is an indispensable parameter for analyzing the earthquake response of site caused by far-field ground. The apparatus used to obtain the dynamic shear modulus is well known as resonant column apparatus. The soil column, installed in this apparatus, is driven by electromagnetic force at free end [9] producing its torsional vibration. If flexural vibration of the soil column occurs, the dynamic Young modulus can be obtained, which is also an important parameter for dynamic analysis of site suffered by near-field ground motion. Essentially, the frequency equation of flexural vibrating cantilever beam with an additional mass is needed. However, the vibrating frequency and shape mode of soil column are effected by not only the shear force but also the moment force, generated by the motion of the additional mass attached at the free end of the soil column. So the influence of these two forces on the vibrating of soil column must be discussed. Cascante et al. [10] derived a frequency equation of this vibrational problem using Rayleigh's method assuming that the mode shape is a third-order polynomial. Laura [11] derived the frequency equation of a cantilever beam attaching an additional mass, which is considered as shear force acted on the free end of beam but did not consider the moment force generated by the mass. And recently, Gürgöze [12-14] studies the eigenfrequencies of a cantilever beam carrying a tip mass or spring-mass.

The paper tried to derive the frequency equation of the cantilever beam in order to obtain more accurate solution for the soil column and to analyze the frequency characteristics 


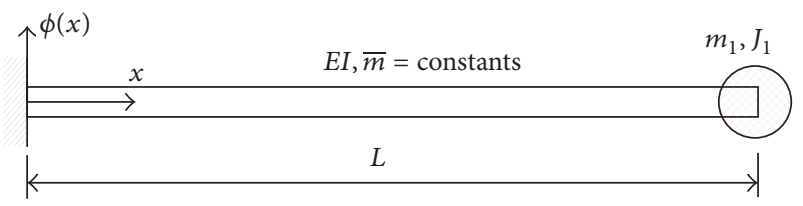

FIGURE 1: Diagram of the cantilever beam with an additional mass.

of this system effected by the bending moment generated by the additional mass.

\section{Flexural Motion Equation of Cantilever Beam with an Additional Mass}

The flexural cantilever beam to be considered is the straight, uniform beam with an additional rigid mass attached by fixed connection. The significant physical properties of this beam are assumed to be the flexural stiffness EI and the mass per unit length $\bar{m}$, both of which are constant along the span $L$. The transverse displacement response $y(x, t)$ is a function of position and time. The free vibration equation of motion for the system as shown in Figure 1 is easily formulated by directly expressing the equilibrium of all forces acting on the differential segment of beam. And it becomes [15]

$$
E I \frac{\partial^{4} y(x, t)}{\partial x^{4}}+\bar{m} \frac{\partial^{2} y(x, t)}{\partial t^{2}}=0 .
$$

Since $E I$ and $\bar{m}$ are constant, one form of solution of this equation can be obtained by separation of variables using

$$
y(x, t)=\phi(x) Y(t)
$$

which indicates that the free vibration motion is of a specific shape $\phi(x)$ having a time-dependent amplitude $Y(t)$. Substituting this equation into (1) can yield two ordinary differential equations.

$$
\begin{aligned}
\ddot{Y}(t)+\omega^{2} Y(t) & =0, \\
\phi^{\prime \prime \prime \prime}(x)-\beta^{4} \phi(x) & =0
\end{aligned}
$$

in which $\beta^{4}=\omega^{2} \bar{m} / E I$, and $\omega$ is the circular frequency. These equations have the solution separately as follows:

$$
\begin{aligned}
& Y(t)=B_{1} \sin (\omega t)+B_{2} \cos (\omega t), \\
& \phi(x)=A_{1} \cos \beta x+A_{2} \sin \beta x+A_{3} \operatorname{ch} \beta x+A_{4} \operatorname{sh} \beta x
\end{aligned}
$$

in which constants $B_{1}$ and $B_{2}$ depend upon the initial displacement and velocity conditions; and real constants $A_{i}$ must be evaluated so as to satisfy the known boundary conditions at the ends of the beam.

The cantilever beam considered has a fixed end, so its two known boundary conditions are

$$
\begin{aligned}
\phi(0) & =0, \\
\phi^{\prime}(0) & =0 .
\end{aligned}
$$

Making use of (5) and its first partial derivative with respect to $x$, from (6) one obtains $A_{3}=-A_{1}$ and $A_{4}=-A_{2}$, So (5) becomes

$$
\phi(x)=A_{1}(\cos \beta x-\operatorname{ch} \beta x)+A_{2}(\sin \beta x-\operatorname{sh} \beta x) .
$$

An additional rigid mass $m_{1}$ having a rotary mass moment of inertia $J_{1}$ is attached by fixed connection to its free end as also shown in Figure 2. These internal force components are along with the translational and rotary inertial force components $m_{1} \omega^{2} \phi(L)$ and $J_{1} \omega^{2} \phi^{\prime}(L)$, respectively. So the force and moment equilibrium of the additional mass requires the boundary conditions

$$
\begin{aligned}
& M(L)=E I \phi^{\prime \prime}(L)=-\omega^{2} \phi^{\prime}(L) J_{1}, \\
& V(L)=E I \phi^{\prime \prime \prime}(L)=-\omega^{2} \phi(L) m_{1} .
\end{aligned}
$$

Making use of (5) and its first, second, and third partial derivative with respect to $x$ and substituting them into (8) yield

$$
\left[\begin{array}{ll}
\beta^{2}(\cos \beta L+\operatorname{ch} \beta L)+\frac{\omega^{2} J_{1}}{E I} \beta(\sin \beta L+\operatorname{sh} \beta L) & \beta^{2}(\sin \beta L+\operatorname{sh} \beta L)-\frac{\omega^{2} J_{1}}{E I} \beta(\cos \beta L-\operatorname{ch} \beta L) \\
\beta^{3}(\sin \beta L-\operatorname{sh} \beta L)+\frac{\omega^{2} m_{1}}{E I}(\cos \beta L-\operatorname{ch} \beta L) & -\beta^{3}(\cos \beta L+\operatorname{ch} \beta L)+\frac{\omega^{2} m_{1}}{E I}(\sin \beta L-\operatorname{sh} \beta L)
\end{array}\right]\left\{\begin{array}{l}
A_{1} \\
A_{2}
\end{array}\right\}=\left\{\begin{array}{l}
0 \\
0
\end{array}\right\} .
$$

For coefficients $A_{1}$ and $A_{2}$ to be nonzero, the determinant of the square matrix in this equation must equal zero, thus giving the frequency equation

$$
\begin{aligned}
& {\left[\beta^{2}(\cos \beta L+\operatorname{ch} \beta L)+\frac{\omega^{2} J_{1}}{E I} \beta(\sin \beta L+\operatorname{sh} \beta L)\right]} \\
& \cdot\left[-\beta^{3}(\cos \beta L+\operatorname{ch} \beta L)+\frac{\omega^{2} m_{1}}{E I}(\sin \beta L-\operatorname{sh} \beta L)\right]
\end{aligned}
$$

$$
\begin{aligned}
& -\left[\beta^{2}(\sin \beta L+\operatorname{sh} \beta L)-\frac{\omega^{2} J_{1}}{E I} \beta(\cos \beta L-\operatorname{ch} \beta L)\right] \\
& \cdot\left[\beta^{3}(\sin \beta L-\operatorname{sh} \beta L)+\frac{\omega^{2} m_{1}}{E I}(\cos \beta L-\operatorname{ch} \beta L)\right] \\
& =0
\end{aligned}
$$






FIGURE 2: Diagram of force equilibrium analysis at the free end.

which can reduce to the following form making use of $\omega^{2} / E I=\beta^{4} / \bar{m}$

$$
\begin{aligned}
& \frac{J_{1} m_{1}}{\bar{m}^{2}} \beta^{4}(1-\cos \beta L \operatorname{ch} \beta L) \\
& +\frac{m_{1}}{\bar{m}} \beta(\sin \beta L \operatorname{ch} \beta L-\cos \beta L \operatorname{sh} \beta L) \\
& =\frac{J_{1}}{\bar{m}} \beta^{3}(\sin \beta L \operatorname{ch} \beta L+\cos \beta L \operatorname{sh} \beta L) \\
& \quad+(1+\cos \beta L \operatorname{ch} \beta L) .
\end{aligned}
$$

Setting

$$
z=\beta L=\sqrt[4]{\frac{\omega^{2} \bar{m}}{E I}} L
$$

is a power function of the frequency of the flexural vibrating cantilever beam. And setting $\alpha_{J}=J_{1} / \bar{m} L^{3}=J_{1} / m_{T} L^{2}$ is a ratio of the rotary mass moment of inertia for the additional mass and for a rigid mass of $m_{T}$ having a rotary arm length of $L$; and $\alpha_{m}=m_{1} / \bar{m} L=m_{1} / m_{T}$ is a ratio of the mass between the additional mass and the cantilever beam. So the frequency equation becomes

$$
\begin{gathered}
\alpha_{J} \alpha_{m} z^{4}(1-\cos z \operatorname{ch} z)+\alpha_{m} z(\sin z \operatorname{ch} z-\cos z \operatorname{sh} z) \\
=\alpha_{J} z^{3}(\sin z \operatorname{ch} z+\cos z \operatorname{sh} z)+(1+\cos z \operatorname{ch} z) .
\end{gathered}
$$

This frequency equation is a transcendental equation that contained two parameters with unambiguous physical meaning. The solution of this equation, which is the frequency of the system of the flexural vibrating cantilever beam, can be only obtained by numerical method for now.

\section{Solution of the Frequency Equation}

In order to solve the frequency equation, the term of the right side of (13) can be moved to the left side, and then suppose the left side equals $f\left(z, \alpha_{J}, \alpha_{m}\right)$. And $f=0$ is equivalent to (13). Suppose the parameters $\alpha_{J}$ and $\alpha_{m}$ are constant, and then the relationship between $f(z)$ and $z$ can be calculated and drawn as shown in Figure 3, in which the parameters $\alpha_{J}=10$ and $\alpha_{m}=1$. However, the value of $f(z)$ rises abruptly. So the $y$ axis alters to $\log f(z)$ in order to show the curve is up and down across the $x$-axis obviously. The intersection points of

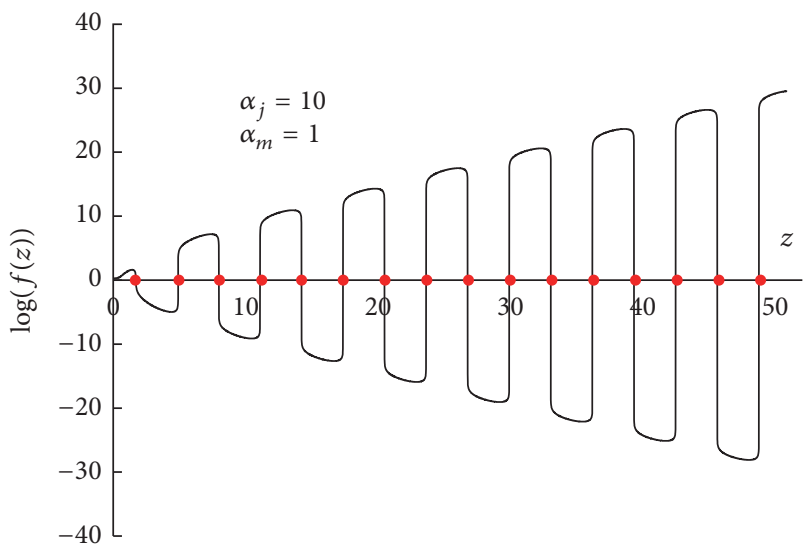

Figure 3: Relationship between $f(z)$ and $z$.

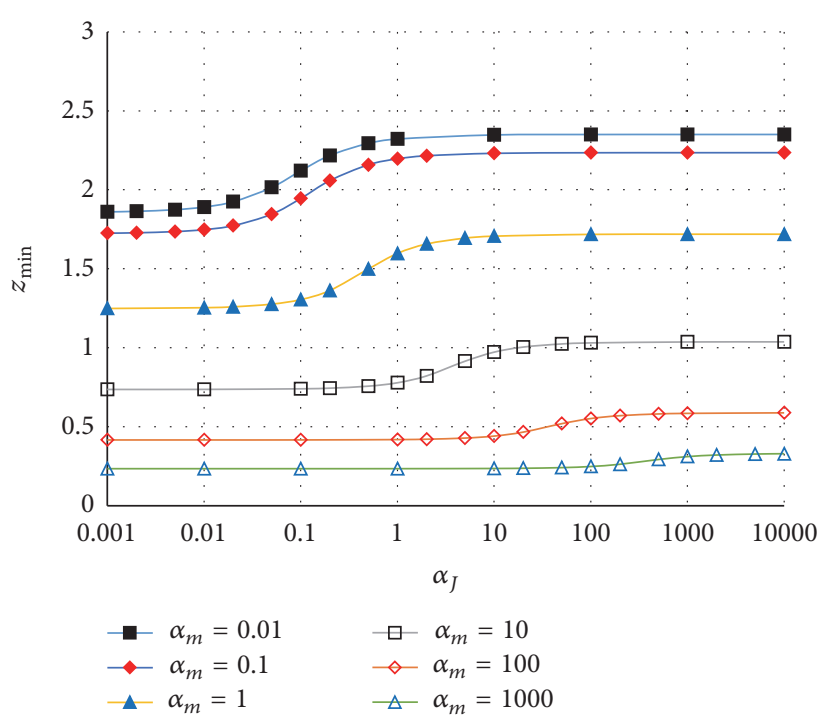

Figure 4: Varied $z$ with $\alpha_{J}$.

the curve and $x$-axis as marked in Figure 3 with circle points are the roots of the equation $f(z)=0$, where its approximate roots can be obtained using numerical methods for solving system of nonlinear equations, such as method of bisection and Newton's method.

For a specific system, where the $\alpha_{J}$ and $\alpha_{m}$ are determined, the solution of the frequency equation (13) can be obtained. And the roots of the parameter $z$ are varied with the parameters $\alpha_{J}$ and $\alpha_{m}$. Figures 4 and 5 show their effects. With the increment of the parameter $\alpha$, the root $z$ is increased identically with a sigmoid function as shown in Figure 4. It means that the frequency of the system will increase if the rotary mass moment of inertia for the additional mass is increased. And the rate of the increment is larger around $\alpha_{J}=\alpha_{m}$ than others. For example, the increment of $z$ at the range of $\alpha_{J}=0.1 \sim 10$ is fastest while $\alpha_{m}=1$. With the increment of the parameter $\alpha_{m}$, the root $z$ is decreasing, as shown in Figure 5, which means that the frequency of the system will decrease with the additional mass increasing. 
TABLE 1: The first five natural frequencies of the cantilever beam considering the bending moment.

\begin{tabular}{lcccccccccc}
\hline \multirow{2}{*}{$\begin{array}{l}\text { Frequency } \\
\text { number (i) }\end{array}$} & $\alpha_{m}=100$ & $\alpha_{m}=10$ & $\alpha_{m}=1$ & $\alpha_{m}=1$ & $\alpha_{m}=1$ & $\alpha_{m}=1$ & $\alpha_{m}=1$ & $\alpha_{m}=1$ & $\alpha_{m}=1$ & $\alpha_{m}=1$ \\
& $\alpha_{J}=1$ & $\alpha_{J}=1$ & $\alpha_{J}=100$ & $\alpha_{J}=10$ & $\alpha_{J}=1$ & $\alpha_{J}=0.1$ & $\alpha_{J}=0.01$ & $\alpha_{J}=0.001$ & $\alpha_{J}=0$ & $(\mathrm{ref}[11])$ \\
\hline 1 & 0.1749603 & 0.6054297 & 2.950242 & 2.91158 & 2.55044 & 1.70226 & 1.571098 & 1.55867 & 1.557298 & 1.557298 \\
2 & 22.30366 & 22.47413 & 23.93841 & 23.93129 & 23.86031 & 23.17405 & 19.13831 & 16.57872 & 16.25009 & 16.25009 \\
3 & 61.66046 & 61.83822 & 63.43232 & 63.42955 & 63.40190 & 63.1286 & 60.72695 & 53.50208 & 50.89584 & 50.89584 \\
4 & 120.90684 & 121.0850 & 122.7265 & 122.7251 & 122.71062 & 122.5668 & 121.2014 & 113.2826 & 105.1983 & 105.1983 \\
5 & 199.86943 & 200.0480 & 201.7208 & 201.71991 & 201.71107 & 201.6229 & 200.7639 & 194.2422 & 179.2320 & 179.2320 \\
\hline
\end{tabular}

${ }^{*}$ Notes. The values of this column were calculated by authors using the frequency equation from [11].

And the results were carefully checked by comparing the values of $z_{i}$ (using the character $y_{i}$ in this reference).

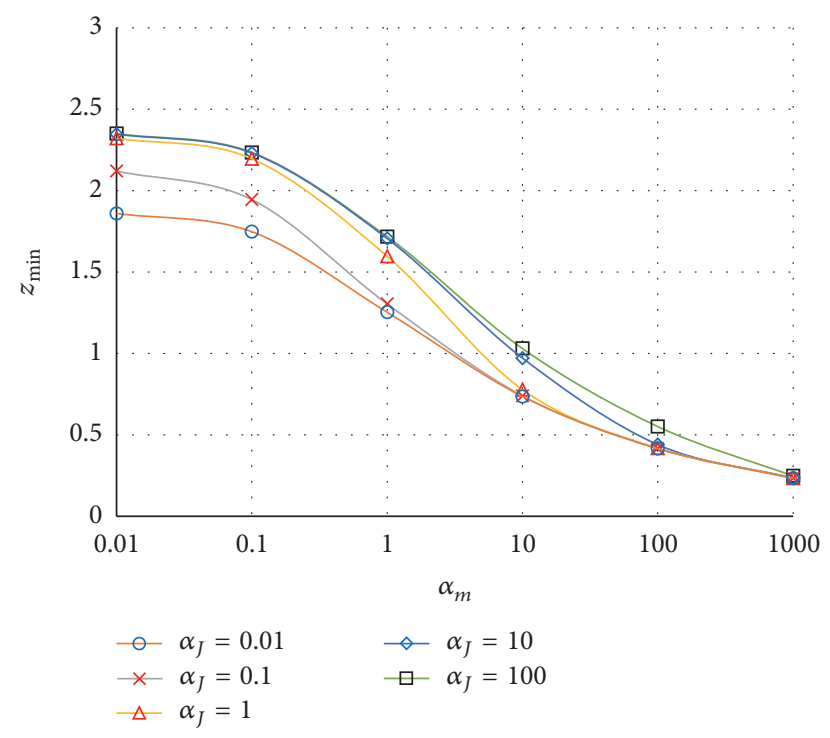

FIGURE 5: Varied $z$ with $\alpha_{m}$.

\section{Natural Frequencies and Shape Modes Characteristics}

The natural frequency $\omega_{i}$ of the flexural vibration of cantilever beam considering the bending moment generated by the attached mass can be calculated using the transformation of (12). Table 1 lists the results of the first five natural frequencies of this system with the varied parameters values $\alpha_{j}$ and $\alpha_{m}$. The natural frequency increases with the parameter $\alpha_{m}$ decreasing, which means the quality of the additional mass reduction compared to that of the cantilever beam. This is consistent with the result of others $[7,16]$. And the frequency also increases with the increment of the rotary mass moment of inertia for the additional mass compared to the beam, which is defined as the parameter $\alpha_{j}$. In order to make comparisons, the results of frequencies when the parameters $\alpha_{j}=0$ and $\alpha_{m}=1$ are also listed, which means the free end of beam has no moment force generated by the additional mass. As expected, these natural frequency values are the same as that obtained from [11], in which only the shear force is considered generated by the attached mass. And the larger value of $\alpha_{j}$ causes the higher frequency of the cantilever beam as shown in Table 1.

However, the interval between the two frequencies for $\alpha_{j}$ equal to zero and for $\alpha_{j}$ unequal to zero is considerable, especially that between the two higher frequency numbers. So the ignored rotary mass moment of inertia may be too ideal for engineering problem and cause obviously error.

The vibration shape mode can also be obtained by substituting the ratio of $A_{1}$ and $A_{2}$ into the shape equation (7). And Figure 6(a) shows the first four mode shapes of the cantilever beam considering the moment force with the parameters $\alpha_{m}=1$ and $\alpha_{J}=10$. The first four mode shapes of the cantilever beam are shown without considering the moment force in [11]. It is clearly shown that the vibration shape is effected by the moment force from the additional mass significantly. However, the existence of the additional mass restrains the development of displacement at the free end of beam.

In order to analyze the influence of $\alpha_{J}$ on the mode shape, the 3rd to 5th mode shapes of considering the moment force with $\alpha_{m}=1$ and $\alpha_{J}=0.001$ and without considering situation are given, as shown in Figure 6(b). Even if the parameter $\alpha_{J}$ is of very small value, the 5 th mode shape of these two situations has obviously divergence, though the smaller the serial number of mode shape is, the less obvious the divergence becomes.

\section{Conclusion}

The frequency equation of cantilever beam with an additional mass exciting flexural vibration was derived considering the rotary inertial moment of inertia of an attached mass, including the shear force. It is a transcendental equation, and it contains two parameters with unambiguous physical meaning, which can be defined as the ratio of rotary mass moment of inertia and the ratio of the mass, respectively.

These two parameters effect both the natural frequency and the shape mode of the beam. As the ratio of rotary mass moment of inertia increases, the natural frequency climbs. Even a little increment of the ratio may cause higher variance between considering and not considering the rotary mass moment of inertia, especially for the high natural frequency. And the ratio of the rotary mass moment of inertia also 


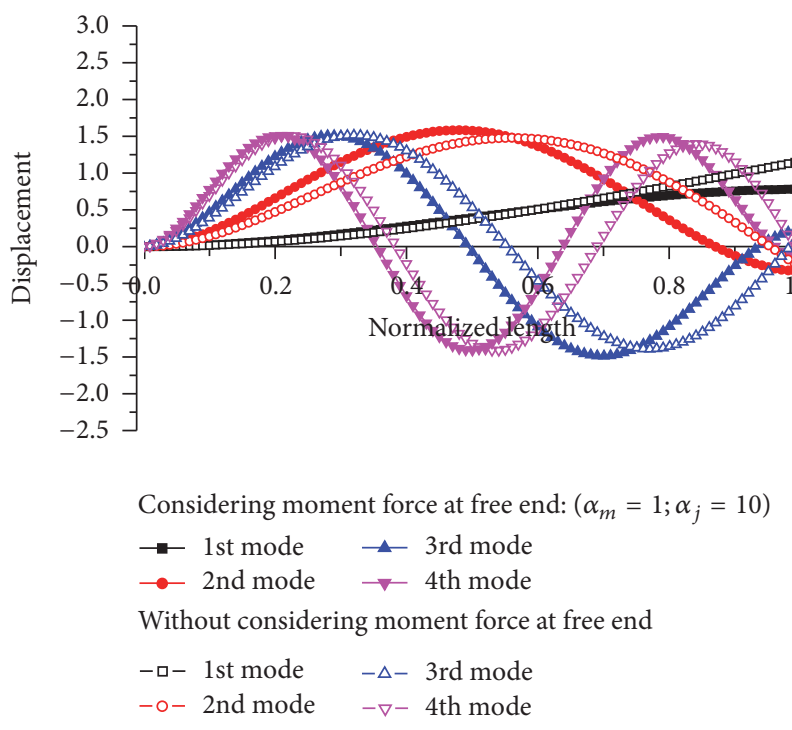

(a)



Considering moment force at free end: $\left(\alpha_{m}=1 ; \alpha_{j}=0.001\right)$

$-\Delta-$ 3rd mode $\quad-\diamond-5$ th mode

$-\nabla-4$ th mode

Without considering moment force at free end

$\rightarrow$ 3rd mode

$\neg \quad 4$ th mode

(b)

Figure 6: The first five mode shapes of considering the moment force with $\alpha_{m}=1$ and $\alpha_{I}=10$ and without considering situation (a) and the 3rd to 5th mode shapes of considering the moment force with $\alpha_{m}=1$ and $\alpha_{J}=0.001$ and without considering situation (b).

effects the mode shape of this system. The higher the serial number of mode shape investigated is, the more obvious the divergence becomes.

\section{Competing Interests}

There are no competing interests regarding this paper.

\section{Acknowledgments}

This paper is supported by National Science Foundation of China (NSFC 51309121) and Natural Science Foundation of Jiangsu Province (BK20130463).

\section{References}

[1] S. S. Rao, Mechanical Vibrations, Pearson Education, Upper Saddle River, NJ, USA, 4th edition, 2004.

[2] Y. K. Rudavskii and I. A. Vikovich, "Forced flexural-andtorsional vibrations of a cantilever beam of constant cross section," International Applied Mechanics, vol. 43, no. 8, pp. 912923, 2007.

[3] D. Zhou and T. Ji, "Estimation of dynamic characteristics of a spring-mass-beam system," Shock and Vibration, vol. 14, no. 4, pp. 271-282, 2007.

[4] D. Zhou and T. Ji, "Dynamic characteristics of a beam and distributed spring-mass system," International Journal of Solids and Structures, vol. 43, no. 18-19, pp. 5555-5569, 2006.

[5] C. A. Rossit and P. A. A. Laura, "Free vibrations of a cantilever beam with a spring-mass system attached to the free end," Ocean Engineering, vol. 28, no. 7, pp. 933-939, 2001.

[6] J. R. Banerjee, "Free vibration of beams carrying springmass systems-a dynamic stiffness approach," Computers and Structures, vol. 104-105, pp. 21-26, 2012.
[7] R. M. Digilov and H. Abramovich, "Flexural vibration test of a beam elastically restrained at one end: a new approach for Young's modulus determination," Advances in Materials Science and Engineering, vol. 2013, Article ID 329530, 6 pages, 2013.

[8] M. I. Idriss and B. H. Seed, "Seismic response of horizontal soil layers," Journal of the Soil Mechanics and Foundation Division, American Society of Civil Engineers, vol. 94, no. 4, pp. 1003-1031, 1968.

[9] V. P. Drnevich, Resonant-Column Testing_Problems and Solutions, ASTM, Denver, Colo, USA, 1978.

[10] G. Cascante, C. Santamarina, and N. Yassir, "Flexural excitation in a standard torsional-resonant column device," Canadian Geotechnical Journal, vol. 35, no. 3, pp. 478-490, 1998.

[11] P. A. A. Laura, J. L. Pombo, and E. A. Susemihl, "A note on the vibrations of a clamped-free beam with a mass at the free end," Journal of Sound \& Vibration, vol. 37, no. 2, pp. 161-168, 1974.

[12] M. Gürgöze, "On the representation of a cantilevered beam carrying a tip mass by an equivalent spring-mass system," Journal of Sound and Vibration, vol. 282, no. 1-2, pp. 538-542, 2005.

[13] M. Gürgöze, "On the eigenfrequencies of a cantilever beam carrying a tip spring-mass system with mass of the helical spring considered," Journal of Sound and Vibration, vol. 282, no. 3-5, pp. 1221-1230, 2005.

[14] M. Gürgöze, "On the eigenfrequencies of a cantilever beam with attached tip mass and a spring-mass system," Journal of Sound and Vibration, vol. 190, no. 2, pp. 149-162, 1996.

[15] R. Clough and J. Penzien, Dynamics of Structures, McGraw-Hill, 1993.

[16] L. E. Monterrubio, "Free vibration of shallow shells using the Rayleigh-Ritz method and penalty parameters," Proceedings of the Institution of Mechanical Engineers, Part C: Journal of Mechanical Engineering Science, vol. 223, no. 10, pp. 2263-2272, 2009. 


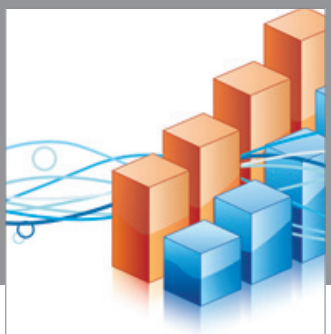

Advances in

Operations Research

vatem alat4

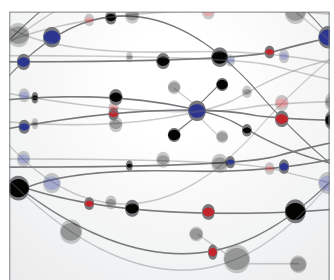

\section{The Scientific} World Journal
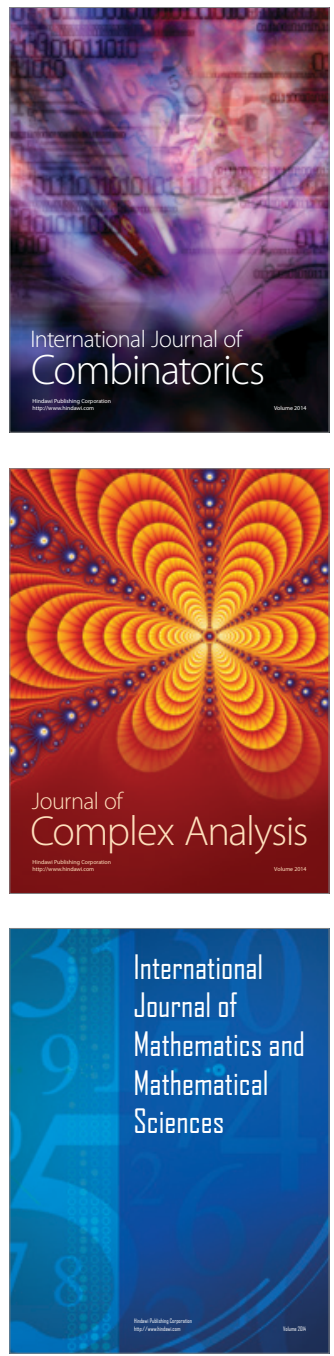
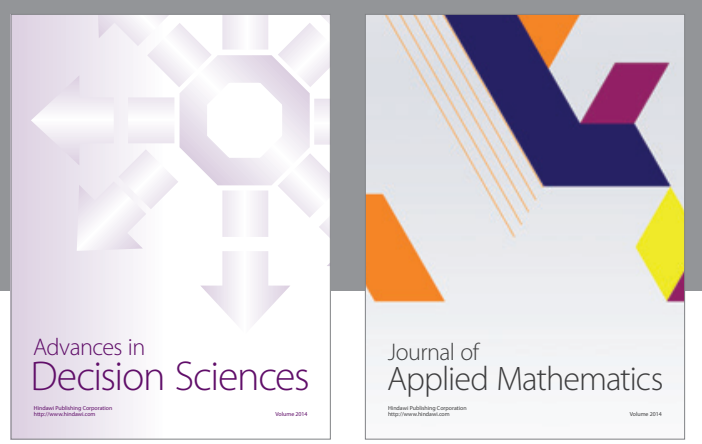

Algebra

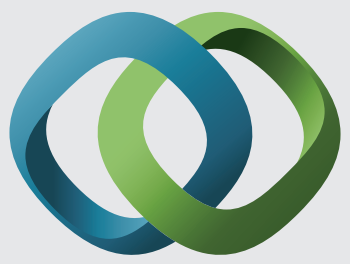

\section{Hindawi}

Submit your manuscripts at

https://www.hindawi.com
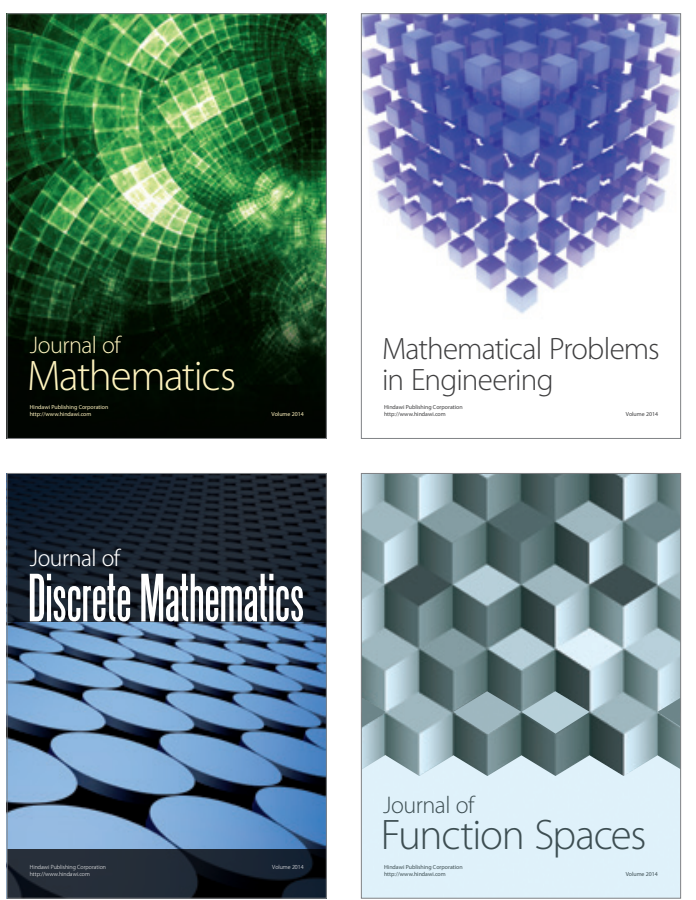

Mathematical Problems in Engineering


Journal of

Function Spaces



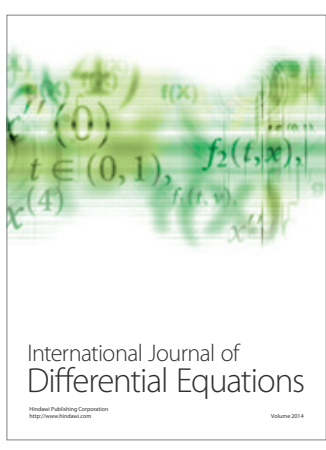
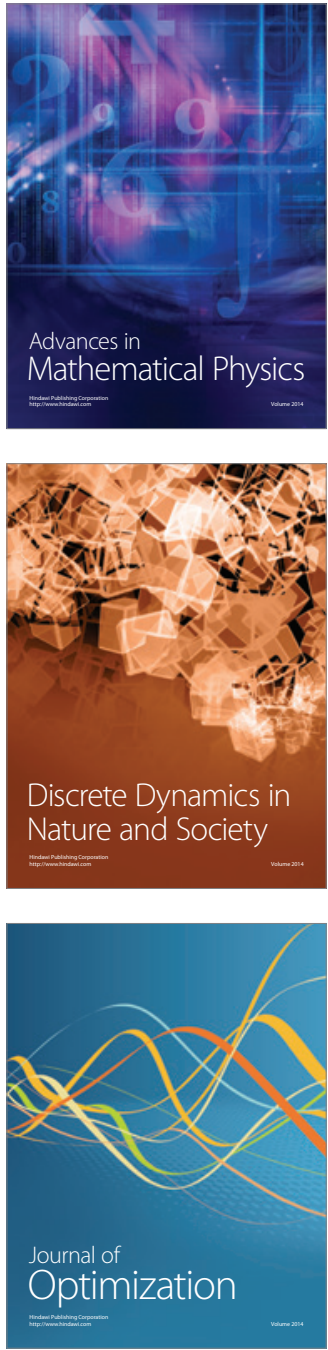